\title{
Reseña \\ Ana María Pérez Rubio y Pablo Barbetti (Coordinadores) (2016) Políticas sociales. Prácticas y significaciones. Bs. As. Estudios Sociológicos Editora, 153pp.
}

Nidia Piñeyro*

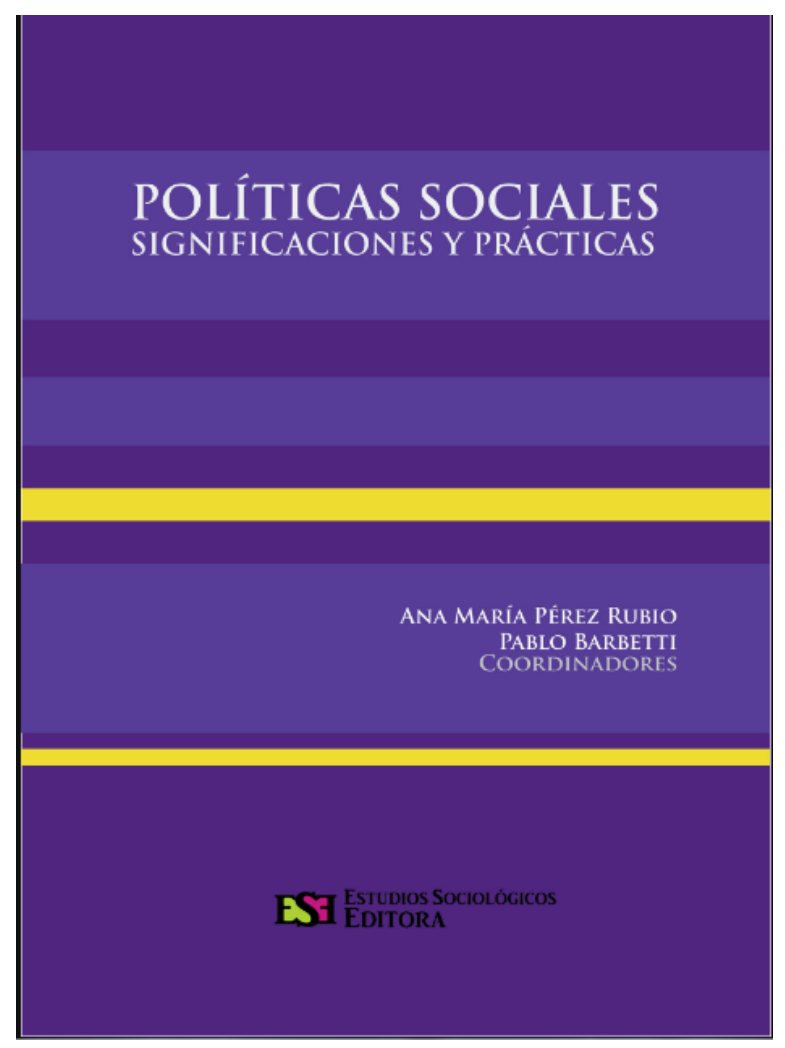

\section{Disponible en:}

http://estudiosociologicos.org/portal/politicas-sociales-significaciones-y-practicas/

\footnotetext{
* Instituto de Investigaciones en Educación, Fac. Humanidades, UNNE. Magíster y Especialista en Desarrollo Social por la Facultad de Humanidades de la Universidad Nacional del Nordeste. Profesora en Letras por la misma institución. Doctoranda en Ciencias Sociales y Humanas por la Universidad Nacional de Misiones. Docente Regular responsable de la Cátedra Introducción a las Ciencias Sociales. Coordinadora del Grupo de Investigación y Desarrollo Estudios Socioculturales de la Región NEA. Miembro académico de la Red WATERLAT-GOBACIT. Correo electrónico: nidiapi@yahoo.com
} 
Una de las claves para leer el texto que estamos recomendando es entender con claridad, como lo hacen la prologuista y los presentadores, una de las tensiones inherentes al campo de estudio: las Políticas Sociales -en adelante PPSS- constituyen una de las líneas de las políticas de Estado que intenta, con distintos matices, mantener el trabajo subordinado al capital, evitando el desbarranque de un sistema social y económico desigual. Pero hay otras líneas, que, aunque no tematizadas, conducen el hilo de la historia explícita: las políticas orientadas a los titulares de la riqueza, el otro polo de la sociedad desigual.

$Y$ esto no es una lectura sobre las políticas del Bicentenario, solamente, sino sobre las PPSS del Estado Moderno. En este sentido, la exploración crítica que hacen los autores de los capítulos, en el momento en el que lo hacen y lo publican, me parece un acto de valentía.

No es fácil trabajar en análisis de políticas en tiempo real. Pareciera que a uno le falta la perspectiva, que le fallaría el extrañamiento. Peor aún si no comulga con el sentido común cotidiano o académico -y ahora, mediático o contra-mediático. Es un camino de aislamiento, exige rigor, prudencia, observables, sobre todo, observables; porque cuando uno está en medio de una atmósfera de euforia por los logros en la promoción de derechos ciudadanos, es muy complicado dar malas noticias, lo que equivale a mitigar el alcance de cualquier programa político homologado a la revolución. El libro desafía con trabajo de campo en todos los niveles, rigurosa revisión teórica y de antecedentes, propuestas metodológicas innovadoras, análisis en diversidad de escalas, actores y programas a los sentidos otorgados por el oficialismo que asocia sin mayores rodeos la gestión Kirchner-Kirchner al nuevo keynesianismo.

El primer capítulo que deseo comentar es el de María Isabel Ortiz y Ana María Pérez Rubio, Problemas Sociales y Políticas Sociales Neo Desarrollistas, Programa Argentina Trabaja. Creo que allí se plasma con claridad el sistema de PPSS del Bicentenario, ubicando con minuciosidad la red de acciones de gobierno. Se problematiza su dimensión territorial a partir de la explicitación de los espacios de gestión, la diversidad de programas, destinatarios, culturas institucionales, modos de comunicación, organización y negociación. Aporta las definiciones necesarias para comprender las tensiones generadas entre la idea de desposesión/igualdad y libertad en los modelos de PPSS que se confrontan, en mayor o menor medida, en todos los demás trabajos.

, a partir de los sentidos otorgados por los funcionarios nacionales y provinciales, y el modo en que fue plasmada en las propuestas y programas. Encuentran que las políticas sociales integrales en territorio ponen el acento en la articulación de recursos técnicos y financieros, actores y espacios de diálogo para superar la fragmentación y fortalecer la identidad nacional. 
Concluyen que, en los hechos, a nivel local, en la provincia de Corrientes, sigue primando el concepto de promoción humana, la perspectiva moral de corte religioso con su consecuente abordaje caritativo-educativo. Es una muestra de cómo, al territorializarse las políticas, la idea de comunidad, clave para el nuevo pensamiento del Ejecutivo nacional, se desdibuja en la definición de los problemas tanto como el modo de reclutamiento y el tipo de gestión. El enfoque de derechos proclamado se diluye en el de atención a los problemas que los funcionarios correntinos suponen adversos a la conservación de la familia y a la inclusión en el mundo del trabajo.

Los entrevistados no incluyen en su discurso las causas por las cuales las personas o comunidades quedan excluidas. Este análisis que deja las causas estructurales de la pobreza en el umbral de las políticas sociales es una constante en los casos que se estudian.

Pablo Barbetti y Federico Butti, en su artículo Programa Ingreso Social con Trabajo. Implementación y Significaciones Construidos por los Sujetos Participantes, por su parte, se proponen revisar cómo funcionan los marcos interpretativos y teóricos que sustentan las propuestas de un programa, y cuáles son los sentidos o significaciones que tienen los mismos para los sujetos participantes. En este capítulo aparece otro rasgo frecuentemente tematizado a lo largo de la obra: la explicitación de la naturaleza construida de las PPSS y de los Programas Sociales, la conceptualización de los mismos como procesos donde se ejercen diferentes mediaciones: capacidades, recursos, intereses. El estudio enmarca la visión de funcionarios, técnicos y destinatarios de políticas de empleo con acento en la consigna "ingreso con trabajo". Para quien no se haya preguntado la diferencia entre trabajo, empleo e ingreso, este capítulo puede ser una caja de malas noticias. En primer lugar, porque expone desde los propios destinatarios la tensión satisfacción/frustración de ser casi un empelado, casi un cooperativista pero, al fin y al cabo, un asistido por un plan social. Lo que rescato del artículo, sin embargo, es de orden teórico, porque lo que pone en la mesa de discusión es una tensión ya problematizada en las ciencias políticas dedicadas al campo laboral: me refiero al análisis que emerge en el cruce de trabajo y política. Es como si preguntaran "¿Quién le va a decir al ciudadano común que la sociedad del contrato salarial o el pleno empleo se terminaron?". Me parece un punto de inflexión para comprender el resto del libro porque da cuenta que, así como es necesario subrayar que el Estado Moderno es pro mercado pero des-mercantilizante a la vez, el empleo para todos y todas es una quimera en la sociedad de la información donde las máquinas y los software han hecho mucho más que aliviarnos de tareas físicas ingratas: nos han hecho perder empleos. Esto, que entre los estudiosos del área está suficientemente consolidado, no permea el discurso político y es entendible: es costosísimo blanquear semejante estatuto. Lo que el artículo revela es que el destinatario sabe perfectamente la diferencia entre lo que significa tener un empleo de 
buena calidad y ser beneficiario de un proyecto, programa o acción específica, donde se recrea la figura del pago con contraprestación de servicios para desasociarlos -y desasociar a la gestión nacional- del concepto de vago/ asistido. Además de lo que acarrea implementar cooperativas sin que medie la voluntad y la claridad en el ejercicio de los roles, el programa Argentina Trabaja es un caso más de intento por parte de las PPSS neo desarrollistas de desmercantilizar un sistema que termina siendo funcional al mercado.

Una lectura más específica, no sobre los casos o programas, sino sobre los enfoques desde los cuales se posicionan los autores de los artículos que componen esta obra colectiva, me sugiere que La Construcción de las Necesidades Habitacionales de las Políticas Públicas como Proceso de Disputa entre Actores, de Venettia Romagnoli, resulta clave para entender el proceso de reconocimiento de las necesidades por parte del Estado y su posterior satisfacción. La perspectiva adoptada considera las "necesidades humanas" como un espacio de disputa entre actores. Esta manera de interpretarlas asume la dimensión política, el carácter conflictivo de un espacio donde pugnan actores con distintas cuotas de poder y con diferentes interpretaciones sobre lo que es necesario. La pregunta que sobrevuela el texto, entonces, excede el campo de las políticas habitacionales, puede ser un interrogante común a cualquier estudio que se plantee visibilizar la totalidad de eventos que configuran un programa, un proyecto, una acción política desde que una necesidad se convierte en demanda hasta la elección de los satisfactores. Las PPSS, desde este enfoque, son entendidas como una serie de reescrituras, un conjunto de procedimientos mediante los cuales las necesidades politizadas se traducen en necesidades administrables.

La conclusión de Romagnoli sugiere que en Políticas Habitacionales aún es una deuda la promoción genuina de participación de actores políticos, económicos y académicos por parte del Estado, cuyo rol es mitigar la asimetría e integrar la dimensión sociocultural de las necesidades sin descuidar el derecho a participar.

En consonancia con estas conclusiones, Mercedes Oraisón, en su artículo La Participación como Estrategia de Inclusión. El Discurso de las Políticas del Bicentenario, advierte la falta de inclusión de los beneficiarios en los procesos de formulación de políticas. En el análisis del discurso oficial, específicamente del Ministerio de Desarrollo de la Nación, no hay menciones de procedimientos o mecanismos en la definición de PPSS; aunque se los reconoce como titulares de derecho en lugar de objetos de protección y se prevén acciones de capacitación, diagnósticos de problemas, cogestión de recursos, se hace dentro de un espacio que deja afuera el cuestionamiento al modelo hegemónico de gestión de PPSS.

la reseña, me gustaría comentar, en primer lugar, el artículo de Socorro Foio y, luego el de Ana María Pérez Rubio.En Espacios de Participación Ciudadana ¿Fortalecimiento de la Democracia o Consolidación del Orden Dominante?, la respuesta demora en 
aparecer; demora porque es una respuesta metodológica, fundamentada, compleja, casi exquisitamente.

Para contestarla, la autora se vale de un cuerpo de conceptos de variada raigambre teórica (Teoría de sistemas, Teoría de los signos, Teoría del aprendizaje, Teoría de las organizaciones) que le permiten esquivar la dupla heteronomía/autonomía como indicador privilegiado para describir la calidad de la participación en una institución.

Al utilizar el método dialéctico, imagina que no solo la dupla heterónomo/autónomo es insuficiente, sino que pueden verse como los momentos extremos de un proceso donde caben otros dos.

El aporte de Foio, además de la complejización de esta taxonomía, consiste en ofrecer espacios de observación relacionales y jerárquicos, cuya lectura generarían un valor en el nivel superior. Observados la concurrencia y diversidad de actores, el estilo institucional y el tipo de prácticas, se pueden diferenciar las gestiones que serán caracterizadas por su tendencia al control/cooptación/concertación/autonomía.

De esta suerte, podemos encontrar que las características de cooptación y concertación, tanto como de autonomía o de control, co-existen en todas las gestiones y lo que puede operar como pasaje entre un estilo y otro son prácticas concretas, en niveles concretos y actores concretos. La respuesta metodológica, entonces, deriva en una recomendación de política. Si nos interesa fomentar la participación, lo que podemos empezar por entender es que las organizaciones son organismos que aprenden.

Por último, en su artículo Las Políticas Sociales como Instituciones Totales. La Construcción de Subjetividad, Ana María Pérez Rubio subraya la intencionalidad de las Políticas del Bicentenario de inaugurar un camino que recupere el espacio comunitario, la reconstrucción del tejido social, la disminución de la pobreza y la desigualdad al propiciar la inclusión por recurso al fortalecimiento de la autonomía y el protagonismo de los involucrados.

Analiza dos Programas del Bicentenario, Argentina Trabaja y Asignación Universal por Hijo. Las preguntas que orientan el estudio se relacionan con la incidencia de las PPSS en la producción de sujetos y en qué medida generan, favorecen y fortalecen proyectos de autonomía que vayan más allá de garantizar el orden, sin alterar el funcionamiento de la economía y la sociedad que producen la exclusión.

Lo que destaca en el trabajo de Pérez Rubio, es la riqueza que extrae de la diferenciación entre los conceptos de subjetividad y subjetivación. Mientras la primera implica la integración de la memoria, las prácticas sociales, el pensamiento, las representaciones sociales producidos por los individuos a partir de su conciencia y su cultura, la segunda es el proceso por el cual un individuo asume su singularidad. En tal sentido, dice, no hay procesos uniformes de subjetivación; sin embargo, podemos pensar en ciertos mecanismos de integración social que colaboran con la asunción de ciertos rasgos de individuación negativa. 
Si bien Pérez Rubio reconoce que la convocatoria a las organizaciones sociales y comunitarias, el pasaje de beneficiario a sujeto de derecho y la promoción de estrategias asociativas para la economía solidaria son algunos de los cambios que han sido incorporados, los mismos no dejan de constituirse en un proyecto compensatorio que admite como punto de partida la desigualdad social básica; mientras que las acciones asociativas que se propician, orientadas hacia la producción auto-gestionada se ajustan a un conjunto de requerimientos que deben ser satisfechos para cubrir el déficit de inserción, replican, de este modo, la lógica del capitalismo de consumo.

Un aporte a la comprensión de las formas de subjetivación refiere a tres modelos. Un primer modelo se configura a partir de contextos de socialización vinculados directamente con la crisis estructural del empleo, la implementación de políticas sociales compensatorias y condiciones precarias de existencia que definen sus trayectorias vitales en términos de beneficiarios, donde el sujeto asume la disciplina sin cuestionamiento. Este modo, aun cuando sugiere adaptación a la situación, supone en verdad una cierta pasividad más próxima a la sumisión y al conformismo que a la aceptación reflexiva por parte del sujeto.

Cuando la trayectoria reconoce como ámbitos de socialización y experiencia laboral que caracterizaron a la sociedad salarial-, el sujeto tiene la posibilidad de distanciarse, de mirarlo desde otro lado; sin embargo, aparece de modo recurrente la mirada desconfiada con respecto al otro, sea porque no se le da el uso adecuado al ingreso universal, o porque no se cumple con la obligación de trabajar que implica el programa.

Finalmente, hay un tercer tipo, cuya principal característica se encontraría en la no implicación y la prescindencia. La persona busca su incorporación al programa; no obstante, en su comportamiento pone de manifiesto un claro rechazo a ajustarse a las normas establecidas, sin que constituya necesariamente un modo de resistencia. Se trataría de una forma de cuestionar la ideología del orden social con la pretensión de obtener el beneficio, pero sin cumplir con las reglas que disciplinan y ordenan, privilegiando sus finalidades por sobre las finalidades de la institución.

La autora considera que ninguno de estos comportamientos resultaría exitoso con referencia a la autonomía del sujeto, y afirma la existencia de una cierta circularidad entre las situaciones de exclusión y la subjetividad que se conforman, las que favorecen procesos de individualización negativa, entendidos como carencia de seguridad, de consideración, de lazos estables, con el consecuente aislamiento, desconexión y creciente insatisfacción identitaria.

La conclusión es contundente y desalentadora: la implementación de políticas sociales orientadas a disminuir la alteridad social estaría operando más bien como mecanismo para atenuar el conflicto y reducir el sufrimiento. La integración no puede consistir exclusivamente en la mejora de los niveles de vida o el acceso a una mayor cantidad de satisfactores, sino en la creación de capacidad de vida con una lógica de 
ensanchamiento de la subjetividad que exprese la potencialidad del sujeto individual, en lugar de restringirse a la lógica de la reproducción material.

No quisiera cerrar la reseña sin reflexionar en torno a una cuestión, una pregunta que me acompañó en la lectura de estos textos: ¿Qué mediaciones están operando para que una consigna de máxima -tal como aparece en el discurso de las Políticas del Bicentenario- sea sustituida en la comprensión y explicación por una consigna de mínima y hasta opuesta?

Pareciera necesario advertir que este conjunto de políticas reclama para sí una hipótesis de lectura en clave de ruptura y desmarcamiento total respecto de las implementadas en el período inmediatamente anterior; pero cuando son interpeladas desde la contrastación en relación con los estilos institucionales que las atraviesan, los sentidos otorgados por los destinatarios, los efectos logrados, no digamos ya los marcos teóricos -que en política suelen ser fuertemente deontológicos-, lo que prevalece es el desfasaje entre lo proclamado/diseñado y lo implementado/vivido; eso que las muestran, como lo que pudieron ser, en un contexto donde la revolución no es posible. En un co-texto donde el Estado es el garante del funcionamiento del mercado y las PPSS, un producto de la lógica de acumulación del capital. De un Estado pro mercado que ensaya políticas sociales des- mercantilizantes, lo que podemos esperar, estructuralmente, es el mantenimiento del status quo. 